\title{
Efficacy of Administration of an Angiotensin Converting Enzyme Inhibitor for Two Years on Autonomic and Peripheral Neuropathy in Patients with Diabetes Mellitus
}

\author{
Triantafyllos Didangelos, Konstantinos Tziomalos, \\ Charalambos Margaritidis, Zisis Kontoninas, Ioannis Stergiou, Stefanos Tsotoulidis, \\ Eleni Karlafti, Alexandros Mourouglakis, and Apostolos I. Hatzitolios \\ Diabetes Center, First Propedeutic Department of Internal Medicine, Medical School, Aristotle University of Thessaloniki, \\ AHEPA Hospital, Thessaloniki, Greece \\ Correspondence should be addressed to Triantafyllos Didangelos; didang@med.auth.gr
}

Received 13 January 2017; Accepted 21 February 2017; Published 8 March 2017

Academic Editor: Ilias Migdalis

Copyright (C) 2017 Triantafyllos Didangelos et al. This is an open access article distributed under the Creative Commons Attribution License, which permits unrestricted use, distribution, and reproduction in any medium, provided the original work is properly cited.

\begin{abstract}
Aim. To evaluate the effect of quinapril on diabetic cardiovascular autonomic neuropathy (CAN) and peripheral neuropathy (DPN). Patients and Methods. Sixty-three consecutive patients with diabetes mellitus [43\% males, 27 with type 1 DM, mean age 52 years (range 22-65)], definite DCAN [abnormal results in 2 cardiovascular autonomic reflex tests (CARTs)], and DPN were randomized to quinapril $20 \mathrm{mg} /$ day (group A, $n=31$ ) or placebo (group B, $n=32$ ) for 2 years. Patients with hypertension or coronary heart disease were excluded. To detect DPN and DCAN, the Michigan Neuropathy Screening Instrument Questionnaire and Examination (MNSIQ and MNSIE), measurement of vibration perception threshold with biothesiometer (BIO), and CARTs [R-R variation during deep breathing [assessed by expiration/inspiration ratio (E/I), mean circular resultant (MCR), and standard deviation (SD)], Valsalva maneuver (Vals), $30: 15$ ratio, and orthostatic hypotension $(\mathrm{OH})$ ] were used. Results. In group A, E/I, MCR, and SD increased ( $p$ for all comparisons < 0.05). Other indices (Vals, $30: 15, \mathrm{OH}, \mathrm{MNSIQ}, \mathrm{MNSIE}$, and BIO) did not change. In group B, all CART indices deteriorated, except Vals, which did not change. MNSIQ, MNSIE, and BIO did not change. Conclusions. Treatment with quinapril improves DCAN (mainly parasympathetic dysfunction). Improved autonomic balance may improve the long-term outcome of diabetic patients.
\end{abstract}

\section{Introduction}

Diabetes mellitus (DM) is the most common cause of neuropathy and diabetic neuropathy $(\mathrm{DN})$ comprises a heterogeneous group of disorders that can cause neuronal dysfunction throughout the human body. The Toronto Consensus Panel on DN divided in 2010 the disease into typical and atypical neuropathy [1] Typical diabetic peripheral neuropathy (DPN) is "a symmetrical, length-dependent sensorimotor polyneuropathy attributable to metabolic and microvascular alterations as a result of chronic hyperglycemia exposure and cardiovascular risk covariates." Atypical forms of DN differ in onset, course, manifestations, associations, and putative mechanisms and are likely to be associated with pain and/or dysautonomia. Peripheral and autonomic neuropathies are the most common manifestations of DN, which often coexist. Diabetic peripheral neuropathy is the second most common form of DN and is estimated to affect $45-50 \%$ of all patients with DM [2]. The prevalence varies according to the severity and duration of hyperglycaemia but overall polyneuropathy is present in up to $50 \%$ of people with long-standing DM [3].

DPN represents a major health problem as it may present with excruciating neuropathic pain and is responsible for substantial morbidity, resulting from foot ulceration, amputations, and impaired quality of life, as well as with increased mortality. The manifestations of diabetic autonomic neuropathy (DAN) are manifold affecting all systems and organs innervated from autonomic system, but cardiovascular, urogenital, gastrointestinal, pupillomotor, thermoregulatory, and sudomotor systems are the most important. Diabetic 
TABLE 1: Characteristics of patients at baseline.

\begin{tabular}{|c|c|c|c|}
\hline & $\begin{array}{c}\text { Group A } \\
(n=31)\end{array}$ & $\begin{array}{l}\text { Group B } \\
(n=32)\end{array}$ & $p$ \\
\hline Age (years) & $52.7 \pm 16.4$ & $51.9 \pm 13.9$ & NS \\
\hline Males (\%) & 48.4 & 37.5 & NS \\
\hline Diabetes mellitus duration (years) & $17.8 \pm 7.4$ & $18.1 \pm 8.2$ & NS \\
\hline Type 1 diabetes mellitus (\%) & 45.2 & 40.6 & NS \\
\hline $\mathrm{HbA}_{1 \mathrm{c}}(\%)$ & $7.1 \pm 2.2$ & $7.2 \pm 2.4$ & NS \\
\hline Fasting plasma glucose (mg/dl) & $125 \pm 27$ & $129 \pm 19$ & NS \\
\hline Total cholesterol (mg/dl) & $189 \pm 37$ & $193 \pm 51$ & NS \\
\hline High density lipoprotein cholesterol (mg/dl) & $46 \pm 10$ & $45 \pm 10$ & NS \\
\hline Low density lipoprotein cholesterol (mg/dl) & $107 \pm 36$ & $118 \pm 49$ & NS \\
\hline Triglycerides (mg/dl) & $185 \pm 70$ & $171 \pm 68$ & NS \\
\hline Creatinine (mg/dl) & $0.97 \pm 0.19$ & $0.98 \pm 0.25$ & NS \\
\hline Estimated glomerular filtration rate $\left(\mathrm{ml} / \mathrm{min} / 1.73 \mathrm{~m}^{2}\right)$ & $112 \pm 34$ & $109 \pm 37$ & NS \\
\hline Uric acid (mg/dl) & $5.4 \pm 1.3$ & $6.1 \pm 2.1$ & NS \\
\hline
\end{tabular}

cardiovascular autonomic neuropathy (DCAN) is characterized by autonomic dysfunction of the cardiovascular system. It is the most prevalent and well-studied form of DAN [4]. It is characterized by alterations in the control of heart rate and vascular hemodynamics. The prevalence of DCAN ranges from 2.5 to $50 \%$ in different cohorts. The prevalence of confirmed DCAN is around $20 \%$ and rises up to $65 \%$ with age and DM duration. DCAN has been shown to negatively impact mortality due to its relationship with serious comorbidities (including silent myocardial ischemia, coronary heart disease (CHD), stroke, diabetic nephropathy, and increased perioperative morbidity) [4]. Thus, the management of DCAN has important implications for the prognosis of DM.

Despite the significant individual and social burden associated with diabetic neuropathy, its treatment remains unsatisfactory. This is in part due to the innately unpredictable and complex nature of the disease, combined with limited systematic diagnostic testing, which differs from diabetic retinopathy and nephropathy, where the disease is more predictable and the diagnostic tests more straightforward. In the current study, we chose to use the most valid and accurate diagnostic tests [Michigan Neuropathy Screening Instrument Questionnaire and Examination (MNSIQ and MNSIE) and Cardiovascular Reflex Tests (CRTs)] for the evaluation of neuropathy in well characterized and highly selected patients. Moreover, there are currently no FDA-approved therapies for diabetic neuropathy and only 3 approved therapies for painful DPN. No treatment results in complete resolution of the underlying pathophysiological abnormalities and treatment of $\mathrm{DN}$ is an unmet need in clinical practice. Only strict metabolic control appears to have a beneficial effect on the prevention and delay of the onset of $\mathrm{DN}$ and to reduce the prevalence of established DN [5-8].

The aim of the present study was to evaluate the efficacy and safety of the administration of an angiotensin converting enzyme (ACE) inhibitor, quinapril $20 \mathrm{mg} /$ day, for two years on DCAN and DPN in patients with type 1 and 2 DM.

\section{Research Design and Methods}

2.1. Patient Selection. This open, parallel-group, controlled study included 63 adult patients with long-standing types 1 and $2 \mathrm{DM}$, who were recruited from the outpatient diabetes clinics in AHEPA University hospital and Hippokration hospital of Thessaloniki, Greece. The study was approved by the institutional ethics committee and all subjects gave written informed consent. The study started in 1999. All patients were asymptomatic, had a normal electrocardiogram, and were normotensive (blood pressure $\leq 130 / 85 \mathrm{mmHg}$ ). They also had normal renal function and were under no medication other than insulin. CHD was excluded on the basis of normal thallium 201 myocardial perfusion imaging. All patients were well characterized and highly selected. Patients were randomized to receive either quinapril $20 \mathrm{mg} /$ day (group A, $n=31$ ) or no treatment (group B, $n=32$ ). Demographic characteristics of the patients are shown in Table 1.

2.2. Cardiovascular Autonomic Reflex Tests (CARTs). The Monitor ONE NDX device (QMED Industries, Clark, NJ, USA) was used for the measurement of the autonomic nervous function (ANF) indices. ANF was assessed according to the consensus statement of the American Diabetes Association and the American Academy of Neurology [9] and the Toronto Consensus Panel on Diabetic Neuropathy [10] taking into account various factors such as concomitant illnesses and lifestyle (exercise, hypoglycemia, smoking, and caffeine intake). The following tests were performed as previously described: (1) beat to beat variation of R-R interval assessed by (a) expiration/inspiration index (E/I Index), (b) mean circular resultant (MCR) vector analysis (probably the most reliable ANF index), and (c) standard deviation (SD) and (2) Valsalva maneuver (Valsalva Index), (3) variation of R$\mathrm{R}$ interval during postural change (30:15 Index), and (4) variation of systolic blood pressure during postural change (standing). The presence of definite DCAN was established if 
TABLE 2: Changes in cardiovascular autonomic reflex tests during the study in patients who received quinapril (group A) and in those who did not (group B).

\begin{tabular}{|c|c|c|c|c|c|c|c|}
\hline & \multicolumn{3}{|c|}{ Group A $(n=31)$} & \multicolumn{3}{|c|}{ Group B $(n=32)$} & \multirow[b]{2}{*}{$\begin{array}{l}p \text { (group A versus } \\
\text { group B at end of } \\
\text { treatment) }\end{array}$} \\
\hline & Baseline & End of treatment & $\begin{array}{c}p \\
\text { (versus } \\
\text { baseline) }\end{array}$ & Baseline & End of treatment & $\begin{array}{c}p \\
\text { (versus } \\
\text { baseline) }\end{array}$ & \\
\hline $\mathrm{E} / \mathrm{I}$ index & $1.11 \pm 0.06$ & $1.23 \pm 0.12$ & 0.011 & $1.09 \pm 0.06$ & $1.04 \pm 0.04$ & 0.007 & $<0.001$ \\
\hline MCR & $18.1 \pm 6.2$ & $38.7 \pm 20.5$ & 0.006 & $14.2 \pm 4.2$ & $8.1 \pm 4.5$ & 0.01 & $<0.001$ \\
\hline SD & $31.1 \pm 11.9$ & $56.6 \pm 23.0$ & 0.004 & $28.2 \pm 9.9$ & $15.5 \pm 7.4$ & $<0.05$ & $<0.001$ \\
\hline Valsalva index & $1.48 \pm 0.28$ & $1.56 \pm 0.33$ & NS & $1.52 \pm 0.22$ & $1.50 \pm 0.28$ & NS & NS \\
\hline $30: 15$ index & $1.15 \pm 0.12$ & $1.18 \pm 0.12$ & NS & $1.15 \pm 0.06$ & $1.08 \pm 0.04$ & $<0.05$ & $<0.001$ \\
\hline $\mathrm{OH}$ & $16.0 \pm 11.8$ & $10.4 \pm 6.1$ & NS & $18.5 \pm 4.5$ & $28.0 \pm 6.3$ & 0.018 & $<0.001$ \\
\hline
\end{tabular}

E/I: expiration/inspiration; MCR: mean circular resultant; SD: standard deviation; OH: orthostatic hypotension.

TABLE 3: Changes in indices of diabetic peripheral neuropathy during the study in patients who received quinapril (group A) and in those who did not (group B).

\begin{tabular}{|c|c|c|c|c|c|c|}
\hline & \multicolumn{3}{|c|}{ Group A $(n=31)$} & \multicolumn{3}{|c|}{ Group B $(n=32)$} \\
\hline & Baseline & $\begin{array}{l}\text { End of } \\
\text { treatment }\end{array}$ & $\begin{array}{c}p \\
\text { (versus } \\
\text { baseline) }\end{array}$ & Baseline & End of treatment & $\begin{array}{c}p \\
\text { (versus } \\
\text { baseline) }\end{array}$ \\
\hline $\begin{array}{l}\text { Michigan Neuropathy } \\
\text { Screening Instrument }\end{array}$ & $2.6 \pm 0.4$ & $2.4 \pm 0.3$ & NS & $2.4 \pm 0.4$ & $2.5 \pm 0.5$ & NS \\
\hline $\begin{array}{l}\text { Vibration perception } \\
\text { threshold }\end{array}$ & $23 \pm 8$ & $20 \pm 7$ & NS & $25 \pm 8$ & $24 \pm 9$ & NS \\
\hline
\end{tabular}

at least 2 of the above-mentioned CARTs were abnormal. The normal values adopted were those reported by Ziegler et al. [11].

2.3. Michigan Neuropathy Screening Instrument (MNSI). MNSI has 2 steps to assess history of neuropathic symptoms and physical examination to evaluate the appearance and sensation of feet. An objective test with 4 questions included foot skin inspection for deformities, dry skin, calluses, infections, fissures and ulcer, ankle reflex, and vibration sensation tested by a $128 \mathrm{HZ}$ tuning fork placed over great toe (MNS1Q). The test was performed by an experienced physician. A score $\geq 2$ was considered abnormal. Abnormality in each item is graded between 0.5 and 1 and at least more than 2 abnormal items are needed to reach the score of neuropathy [12].

All tests were performed in the same day by an experienced physician blinded to the treatment. All patients had both definite DCAN (2 or more CARTs abnormal) and definite DPN.

\section{Statistical Analysis}

All data were analyzed with the statistical package SPSS (version 17.0; SPSS, Chicago, IL, USA). Data are presented as percentages for categorical variables and as mean and standard deviation for continuous variables. Differences in categorical variables between groups at baseline were assessed with the chi-square test. Differences in continuous variables between groups at baseline and at the end of follow-up were assessed with the independent samples $t$-test. Paired samples $t$-test was used for comparisons of DPN and DCAN indices between and after treatment. In all cases, a two-tailed $p<$ 0.05 was considered significant.

\section{Results}

After 2 years of follow-up, improvement was recorded in group $\mathrm{A}$ in all indices of deep breathing test (E/I, MCR, SD) versus baseline (Table 2). The other indices, Valsalva Index, $30: 15$, and orthostatic hypotension did not change versus baseline (Table 2).

In group $\mathrm{B}$, all indices displayed significant deterioration in comparison to baseline at month 24 of follow-up except the Valsalva Index that remained unchanged throughout the study (Table 2).

At the end of follow-up, all indices in group A, except Valsalva Index, were better than in group B (Table 2).

All indices of DPN did not change during the study in either group A or group B and did not differ between the 2 groups at the end of follow-up (Table 3).

\section{Discussion}

The present study demonstrated for the first time that treatment with quinapril for 2 years improves parasympathetic function of DCAN as expressed with the indices of deep breathing test. We did not observe any significant improvement or deterioration in DPN, according to MNSI and BIO indices. 
In the present study, we used CARTs, MNSI, and BIO as the most valid and appropriate tests to diagnose definite neuropathy. This study design was adopted because we wanted to assess the clear effect of ACE inhibition on DCAN and DPN without interference of any other disease except DM or any potential drug-induced change in autonomic nervous system function parameters. Despite the fact that many new methods have been described for the diagnosis of DCAN such as heart rate variability, metaiodobenzylguanidine scan, and corneal confocal microscopy, they are not included in the criteria for the diagnosis of DCAN according to the San Antonio conference and the new proposal from the Toronto panel. The criteria suggested from these 2 conferences are that 2 or more of the following cardiovascular reflex tests should be abnormal: (1) deep breathing test, (2) Valsalva maneuver, (3) $30: 15$ index, and (4) orthostatic hypotension.

The present study included only well characterized, highly selected normotensive patients with definite diagnosed DCAN and DPN free of CHD (based on a normal scintigraphy test), diabetic cardiomyopathy, nephropathy, arrhythmias, or heart failure of any etiology. In our previous study with a 6-month duration, we found an improvement in indices of $24 \mathrm{~h} \mathrm{HRV}$ without any change in indices of CARTs [13]. In another study from our group, we also observed after 1 year of treatment with quinapril improvement of DCAN and left ventricular dysfunction [14]. However, the former study was criticized because of lack of control group. The Steno-2 trial reported that a multifactorial cardiovascular risk intervention (including ACE inhibition) appeared to reduce the prevalence of autonomic dysfunction by 63\% [15]. In the former study, glucose-lowering therapy appeared to have the least impact in preventing DCAN compared with antihypertensive drugs, lipid-lowering agents, antiplatelet therapy, and vitamin and mineral supplementation [15]. In another recent study, patients received a-lipoic acid plus ACE inhibition and there was an improvement in DCAN after 4 years of treatment [16]. Therefore, it is difficult to evaluate the effect of ACE inhibition in the latter two studies, since other treatments were also administered. To the best of our knowledge, the current study is the first that shows the effects of monotherapy with an ACE inhibitor on DCAN.

At baseline, the values of most standard CARTs were below the lower limit of normal. The values of parasympathetic related tests were more adversely affected at baseline than those of sympathetic related tests. It is argued that indices of the deep breathing test (E:I index, SD, and MCR of R-R intervals), considered to be related to vagal tone, were negatively affected and are the earliest markers of DCAN deterioration. So, maybe, they are the first to improve with appropriate treatment, as observed in the current study. Valsalva maneuver is a more complex test; it encompasses a complex reflex arc involving both sympathetic and vagal pathways to the heart, sympathetic pathways to the vascular tree, and baroreceptors in the chest and lungs [11]. Thus, it is reasonable that the Valsalva Index is affected after total and significant autonomic nervous system entanglement, which probably occurs later than the 2 year follow-up period of our study.
Indices of DPN did not change during the 2-year follow-up period. Only 2 randomized, double-blind, placebocontrolled studies evaluated this topic. One trial from Malik et al. reported that peroneal nerve motor conduction velocity increased after 12 months of treatment with trandolapril compared with placebo [17]. Vibration perception threshold, autonomic function and the neuropathy symptom, and deficit score showed no improvement in either group. Our experience with administration of quinapril for 6 months in a randomized, double-blind, placebo-controlled trial was an improvement in indices of $24 \mathrm{~h} \mathrm{HRV}$ with no change in vibration perception threshold. But, in the study by Malik et al., values from indices of CARTs were much lower than in ours and in our study indices of CARTs during 6 months of treatment did not improve and we did not evaluate nerve conduction velocity. The obvious question is why no pathogenetic treatment for DPN has proved sufficiently efficacious to achieve regulatory approval. Ziegler and Luff suggested that trials were hampered by a generally poor design and short follow-up and by being limited to patients with advanced DPN [18]. They suggested that trials involving patients with early DPN, conducted over 3-5 years to establish a delay or arrest in the progression of neuropathy, rather than reversal, were more likely to be successful [18].

Previous studies in experimental models $[19,20]$ have shown a reduction in the progression of DN. These effects are mainly mediated through the vasodilating properties of ACE inhibitors, when used for improvement of nerve flow velocity. A beta blockade-like effect of quinapril was observed in a previous study; that is, quinapril compared with lisinopril decreased the heart rate $(-12 \%, p<0.01)$ in patients with mild to moderate hypertension [21]. In the lisinopril group, no change in heart rate was observed [21]. So, quinapril appears to combine beta blockade-like and ACE inhibition properties, without the side effects of b-blocking agents. This was the reason why we chose to use in the current study quinapril among other ACE inhibitors.

Moreover, formation of advanced glycosylated end products (AGEs) in DM appears to play a crucial role in the pathogenesis of microvascular complications and maybe in the "metabolic memory" observed in large studies. It has been proposed that the pathophysiological cascades triggered by AGEs have a dominant, hyperglycemia-independent role in the onset of the microvascular complications of diabetes [22]. Furthermore, ACE inhibition, in experimental trials, reduces the accumulation of AGEs in DM $[23,24]$ and maybe that is another mechanism of action of these drugs against the development of microvascular complications in DM. Moreover, a beneficial effect of ACE inhibitors has been suggested in many studies on retinopathy and nephropathy.

Data supporting the role of glycemic control in both the primary and secondary prevention of DPN in patients with type $1 \mathrm{DM}$ comes from the Diabetic Control and Complications Trial (DCCT) [5]. In the intensive glucose control arm, a $60 \%$ reduction in the incidence of DPN and a $45 \%$ reduction in the incidence of DCAN were observed [5]. In the Epidemiology of Diabetes Interventions and Complications (EDIC) study, despite no difference in glycemic control, the prevalence and incidence of DPN and CAN were significantly 
reduced in patients who received prior intensive insulin treatment compared with patients who received standard insulin therapy during the DCCT [5]. This protective effect of prior intensive glycemic control, termed metabolic memory, persisted until 13 to 14 years after the end of the DCCT [5]. For CAN, differences in glycated hemoglobin levels during the DCCT explained almost all the protective effects of intensive versus standard therapy on the risk of incident CAN, supporting early commencement of intensive treatment in T1D [5]. In T2DM, there is less evidence of benefits of intensive glycemic control on DN. The United Kingdom Prospective Diabetes Study (UKPDS) emphasized the impact of glycemic control on microvascular complications in type $2 \mathrm{DM}$ and reported a lower rate of impaired vibration perception threshold (VPT) with intensive therapy versus standard therapy, even though this effect became significant only after 15 years of followup (relative risk 0.60, 95\% confidence interval 0.39-0.94) [25]. Furthermore, a Cochrane review of 17 randomized trials concluded that strict glycemic control prevented neuropathy in patients with type $1 \mathrm{DM}$ but a trend towards reduced incidence in type $2 \mathrm{DM}$ was not significant [26].

Our group proposed for the first time the management of DCAN with ACE inhibitors and reported an increase in indices of $24 \mathrm{~h} \mathrm{HRV}$, which have been considered as the earliest markers of autonomic dysfunction [13]. In the present study, we report a clear effect of quinapril in well characterized patients with types 1 and $2 \mathrm{DM}$ with definite DCAN and DPN.

Another drug class that has been studied in the context of DCAN and DPN is aldose reductase inhibitors (ARIs). In a previous study from our group, tolrestat, an ARI, improved indices of CARTs in patients with definite DCAN after 2 years of treatment [27]. In a meta-analysis, $\mathrm{Hu}$ et al. evaluated the efficacy and safety of ARIs for the treatment of CAN in DM, based on CARTs [28]. From their analysis of 10 studies, the authors concluded that ARIs improved cardiac autonomic function [28].

Additional data for the beneficial effect of ACE inhibition on DCAN were reported in the NATHAN-1 trial [16]. The authors used as efficacy measures the Neuropathy Impairment Score of the lower limbs (NIS-LL) and heart rate during deep breathing (HRDB) [16]. Participants treated with alipoic acid for 4 years who received ACE inhibitors showed a greater improvement in HRDB after 4 years [16].

Many other drugs have been used in the management of $\mathrm{DN}$ and further studies are necessary for identifying the best combinations of treatments for diabetic neuropathy [29].

Foot problems from underlying DN are a major cause for developing ulcers, Charcot foot abnormalities, injuries, infections, and lower extremity amputation and this is a lifetime risk for patients with DM. As neuropathy progresses, impairment of body balance and gait abnormalities may be encountered and all these in addition to motor dysfunction may predispose to falls and fractures. Moreover, DCAN could predispose to these adverse events. The economic cost from foot problems is big worldwide and in Greece [30]. Patients with diabetic neuropathy should be routinely counseled about their disease, in particular focusing on patient concerns and expectations. Moreover, the role of strict glycemic control should be emphasized. Thus, patients should be advised on the need for meticulous foot hygiene, appropriate footwear, and mobility support as needed.

In conclusion, in the present study, DCAN (mainly parasympathetic dysfunction) improved after 2 years of treatment with quinapril. Improved autonomic balance may be of clinical importance in the long-term outcome of patients with DM. A clear effect of quinapril on DCAN has been demonstrated. Strict glycemic control is the only confirmed treatment for prevention and delaying the development of diabetic neuropathy today. ACE inhibition and especially quinapril could be an alternative tool for the treatment of $\mathrm{DN}$ and the beneficial effect could be more prominent if the treatment begins at the early stages of neuropathy.

\section{Additional Points}

Novelty Statement. (i) The present study shows the efficacy and safety of treatment with angiotensin converting enzyme (ACE) inhibitors in patients with cardiovascular autonomic neuropathy (CAN), especially in patients with type 2 diabetes mellitus at early stages of the disease, in whom coronary heart disease and hypertension are much more common than in type 1 diabetes mellitus. (ii) Moreover, many studies suffer from inadequate definition of diabetic cardiovascular autonomic neuropathy (DCAN) or supporting data for the presence of DCAN. (iii) The present study explores in highly selected patients this association and is the first to report an improvement in DCAN after treatment with an ACE inhibitor for 2 years.

\section{Ethical Approval}

The study was approved by the Ethics Committee of the Medical School of the Aristotle University of Thessaloniki.

\section{Competing Interests}

The authors have no conflict of interest to declare.

\section{References}

[1] S. Tesfaye, A. J. Boulton, P. J. Dyck et al., "Diabetic neuropathies: update on definitions, diagnostic criteria, estimation of severity, and treatments," Diabetes Care, vol. 33, pp. 2285-2293, 2010.

[2] J. E. Shaw and P. Z. Zimmet, "The epidemiology of diabetic neuropathy," Diabetes Reviews, vol. 7, no. 4, pp. 245-252, 1999.

[3] P. J. Dyck, W. J. Litchy, K. A. Lehman, J. L. Hokanson, J. L. Low, and P. C. O’Brien, "Variables influencing neuropathic endpoints: the rochester diabetic neuropathy study of healthy subjects," Neurology, vol. 45, no. 6, pp. 1115-1121, 1995.

[4] A. I. Vinik and T. Erbas, "Diabetic autonomic neuropathy," Handbook of Clinical Neurology, vol. 117, pp. 27-294, 2013.

[5] R. Pop-Busui, W. H. Herman, E. L. Feldman et al., "DCCT and EDIC studies in type 1 diabetes: lessons for diabetic neuropathy regarding metabolic memory and natural history," Current Diabetes Reports, vol. 10, no. 4, pp. 276-282, 2010.

[6] C. L. Martin, J. W. Albers, and R. Pop-Busui, "Neuropathy and related findings in the diabetes control and complications 
trial/epidemiology of diabetes interventions and complications study," Diabetes Care, vol. 37, no. 1, pp. 31-38, 2014.

[7] I. M. Stratton, A. I. Adler, H. A. W. Neil et al., "Association of glycaemia with macrovascular and microvascular complications of type 2 diabetes (UKPDS 35): prospective observational study," British Medical Journal, vol. 321, no. 7258, pp. 405-412, 2000.

[8] Y. Ohkubo, H. Kishikawa, E. Araki et al., "Intensive insulin therapy prevents the progression of diabetic microvascular complications in Japanese patients with non-insulin-dependent diabetes mellitus: a randomized prospective 6-year study," Diabetes Research and Clinical Practice, vol. 28, no. 2, pp. 103117, 1995.

[9] American Diabetes Association and American Academy of Neurology, "Consensus statement: report and recommendations of the San Antonio Conference on Diabetic Neuropathy," Diabetes Care, vol. 11, pp. 592-597, 1988.

[10] V. Spallone, D. Ziegler, R. Freeman et al., "Cardiovascular autonomic neuropathy in diabetes: clinical impact, assessment, diagnosis, and management," Diabetes/Metabolism Research and Reviews, vol. 27, no. 7, pp. 639-653, 2011.

[11] D. Ziegler, G. Laux, K. Dannehl et al., "Assessment of cardiovascular autonomic function: age-related normal ranges and reproducibility of spectral analysis, vector analysis, and standard tests of heart rate variation and blood pressure responses," Diabetic Medicine, vol. 9, no. 2, pp. 166-175, 1992.

[12] W. H. Herman, R. Pop-Busui, B. H. Braffett et al., "Use of the Michigan Neuropathy Screening Instrument as a measure of distal symmetrical peripheral neuropathy in Typel diabetes: results from the Diabetes Control and Complications Trial/Epidemiology of Diabetes Interventions and Complications," Diabetic Medicine, vol. 29, no. 7, pp. 937-944, 2012.

[13] A. G. Kontopoulos, V. G. Athyros, T. P. Didangelos et al., "Effect of chronic quinapril administration on heart rate variability in patients with diabetic autonomic neuropathy," Diabetes Care, vol. 20, no. 3, pp. 355-361, 1997.

[14] T. P. Didangelos, G. A. Arsos, D. T. Karamitsos, V. G. Athyros, S. D. Georga, and N. D. Karatzas, "Effect of quinapril or losartan alone and in combination on left ventricular systolic and diastolic functions in asymptomatic patients with diabetic autonomic neuropathy," Journal of Diabetes and its Complications, vol. 20, no. 1, pp. 1-7, 2006.

[15] P. Gæde, P. Vedel, N. Larsen, G. V. H. Jensen, H.-H. Parving, and O. Pedersen, "Multifactorial intervention and cardiovascular disease in patients with type 2 diabetes," The New England Journal of Medicine, vol. 348, no. 5, pp. 383-393, 2003.

[16] D. Ziegler, P. A. Low, R. Freeman, H. Tritschler, and A. I. Vinik, "Predictors of improvement and progression of diabetic polyneuropathy following treatment with $\alpha$-lipoic acid for 4 years in the NATHAN 1 trial," Journal of Diabetes and Its Complications, vol. 30, no. 2, pp. 350-356, 2016.

[17] R. A. Malik, S. Williamson, C. Abbott et al., "Effect of angiotensin-converting-enzyme (ACE) inhibitor trandolapril on human diabetic neuropathy: randomised double-blind controlled trial," Lancet, vol. 352, no. 9145, pp. 1978-1981, 1998.

[18] D. Ziegler and D. Luff, "Clinical trials for drugs against diabetic neuropathy: can we combine scientific needs with clinical practicalities?" International Review of Neurobiology, vol. 50, pp. 431-463, 2002.

[19] E. P. Davidson, L. J. Coppey, A. Holmes, and M. A. Yorek, "Effect of inhibition of angiotensin converting enzyme and/or neutral endopeptidase on vascular and neural complications in high fat fed/low dose streptozotocin-diabetic rats," European Journal of Pharmacology, vol. 677, no. 1-3, pp. 180-187, 2012.

[20] L. J. Coppey, E. P. Davidson, T. W. Rinehart et al., "ACE inhibitor or angiotensin II receptor antagonist attenuates diabetic neuropathy in streptozotocin-induced diabetic rats," Diabetes, vol. 55, no. 2, pp. 341-348, 2006.

[21] A. Papageorgiou, A. Karayiannis, V. Athyros, S. Douma, K. Petidis, and C. Zamboulis, "A comparative study of the efficacy and safety of quinapril and lisinopril in patients with mild to moderate hypertension," Drug Investigation, vol. 7, no. 1, pp. 1317, 1994.

[22] N. C. Chilelli, S. Burlina, and A. Lapolla, "AGEs, rather than hyperglycemia, are responsible formicrovascular complications in diabetes: a'glycoxidation-centric' point of view," Nutrition, Metabolism and Cardiovascular Diseases, vol. 23, no. 10, pp. 913919, 2013.

[23] J. M. Forbes, M. E. Cooper, V. Thallas et al., "Reduction of the accumulation of advanced glycation end products by ACE inhibition in experimental diabetic nephropathy," Diabetes, vol. 51, pp. 3274-3282, 2002.

[24] J. M. Forbes, S. R. Thorpe, V. Thallas-Bonke et al., "Modulation of soluble receptor for advanced glycation end products by angiotensin-converting enzyme-1 inhibition in diabetic nephropathy," Journal of the American Society of Nephrology, vol. 16, no. 8, pp. 2363-2372, 2005.

[25] R. R. Holman, S. K. Paul, M. A. Bethel, D. R. Matthews, and H. A. W. Neil, "10-Year follow-up of intensive glucose control in type 2 diabetes," The New England Journal of Medicine, vol. 359, no. 15, pp. 1577-1589, 2008.

[26] B. C. Callaghan, A. A. Little, E. L. Feldman, and R. A. C. Hughes, "Enhanced glucose control for preventing and treating diabetic neuropathy," Cochrane Database of Systematic Reviews, vol. 6, Article ID CD007543, 2012.

[27] T. P. Didangelos, D. T. Karamitsos, V. G. Athyros, and G. I. Kourtoglou, "Effect of aldose reductase inhibition on cardiovascular reflex tests in patients with definite diabetic autonomic neuropathy over a period of 2 years," Journal of Diabetes and its Complications, vol. 12, no. 4, pp. 201-207, 1998.

[28] X. Hu, S. Li, G. Yang, H. Liu, G. Boden, and L. Li, "Efficacy and safety of aldose reductase inhibitor for the treatment of diabetic cardiovascular autonomic neuropathy: systematic review and meta-analysis," PLoS ONE, vol. 9, no. 2, Article ID e87096, 2014.

[29] S. Javed, U. Alam, and R. A. Malik, "Treating diabetic neuropathy: present strategies and emerging solutions," Review of Diabetic Studies, vol. 12, no. 1-2, pp. 63-83, 2015.

[30] I. Migdalis, G. Rombopoulos, M. Hatzikou, C. Manes, N. Kypraios, and N. Tentolouris, "The cost of managing type 2 diabetes mellitus in greece: a retrospective analysis of 10 -year patient level data 'the HERCULES study"' International Journal of Endocrinology, vol. 2015, Article ID 520759, 7 pages, 2015. 


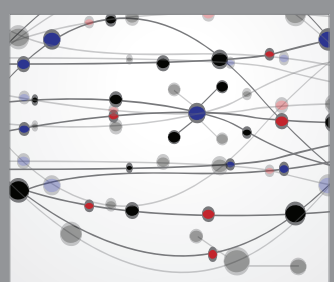

The Scientific World Journal
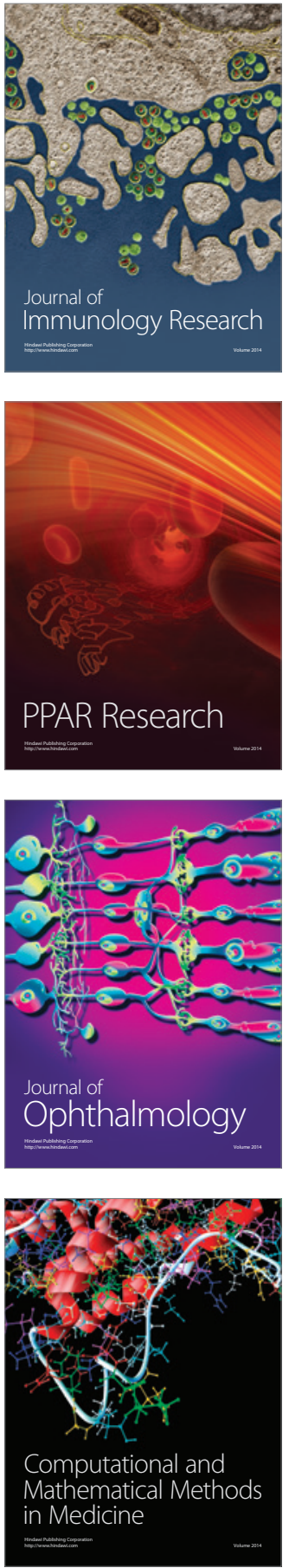

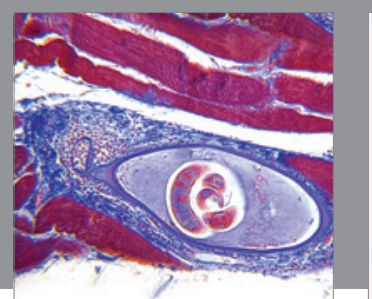

Gastroenterology Research and Practice
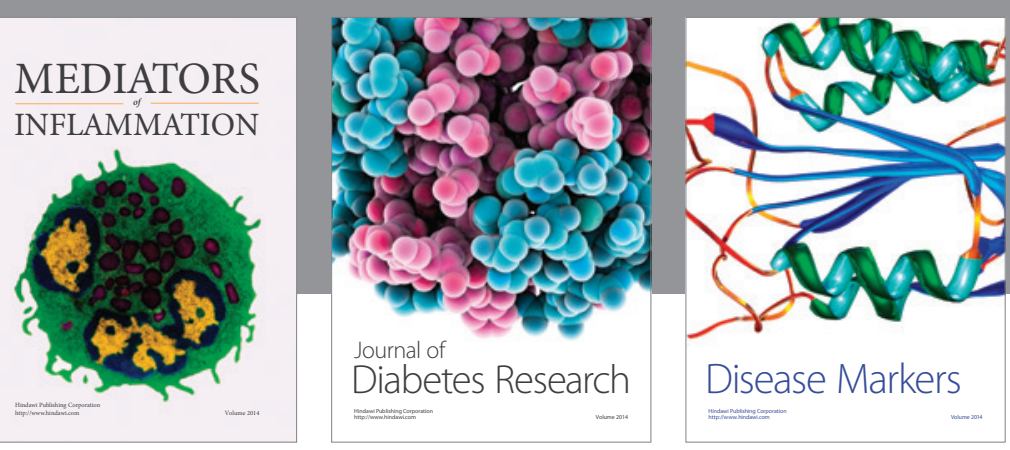

Disease Markers

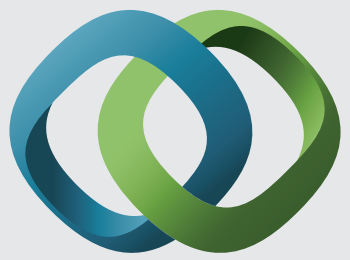

\section{Hindawi}

Submit your manuscripts at

https://www.hindawi.com
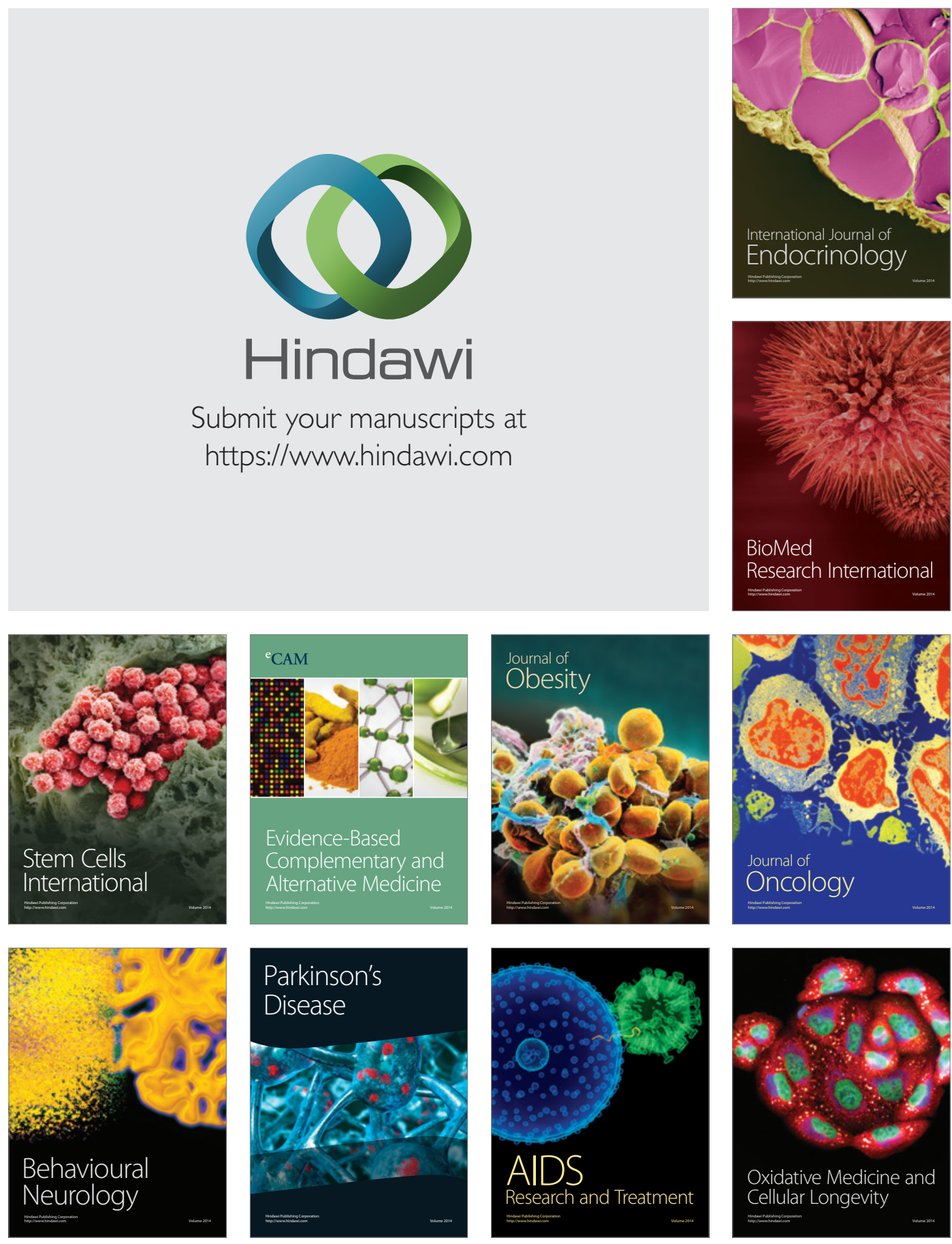